\title{
The Usefulness of Esophageal Baseline Impedance Levels for the Diagnosis of Nonerosive Reflux Disease and the Proper Time for Measurement in Endoscopy-negative Korean Patients With Esophageal or Supraesophageal Symptoms
}

\author{
Young-Gun Kim, Choong-Kyun Noh, and Kwang Jae Lee* \\ Department of Gastroenterology, Ajou University School of Medicine, Suwon,Gyeonggi-do, Korea
}

\section{Background/Aims}

Low baseline impedance levels (BILs) have been suggested to be evidence of GERD. The aim of this study is to investigate the usefulness of esophageal BILs for the diagnosis of nonerosive reflux disease (NERD) and the proper time for measurement in endoscopy-negative Korean patients with esophageal or supraesophageal symptoms.

\section{Methods}

Endoscopy-negative patients with esophageal or supraesophageal symptoms who underwent esophageal multichannel intraluminal impedance-pH monitoring were included. BILs were measured in the proximal and distal esophagus around 10 minutes before meals, 10 minutes and 30 minutes after meals, 30 minutes before the start of nighttime sleep, and 30 minutes and 60 minutes after the start of nighttime sleep.

\section{Results}

A total of 104 patients were included in the study. Distal and proximal esophageal BILs were decreased after meal ingestion. The BILs of the distal esophagus were significantly lower at all time points in the NERD group, but not in the reflux hypersensitivity (RH) group, compared with the functional group. The area under the receiver operating characteristic curve for the diagnosis of NERD was significant at all time points, but that for the diagnosis of RH was not. The cut-off value of $2375 \Omega$ or $2125 \Omega$ measured around 30 minutes before or 60 minutes after the start of nighttime sleep, respectively, were appropriate for the diagnosis of NERD.

\section{Conclusion}

The BILs of the distal esophagus measured at time points before or after the start of nighttime sleep appear to be useful for the diagnosis of NERD, but not for the diagnosis of $\mathrm{RH}$, in endoscopy-negative Korean patients with esophageal or supraesophageal symptoms.

(J Neurogastroenterol Motil 2020;26:463-470)

Key Words

Electric impedance; Esophagus; Gastroesophageal reflux

Received: February 4, 2020 Revised: None Accepted: March 29, 2020

(.) This is an Open Access article distributed under the terms of the Creative Commons Attribution Non-Commercial License (http://creativecommons. org/licenses/by-nc/4.0) which permits unrestricted non-commercial use, distribution, and reproduction in any medium, provided the original work is properly cited.

*Correspondence: Kwang Jae Lee, MD, PhD Department of Gastroenterology, Ajou University Hospital, Ajou University School of Medicine, 164 Worldcup-ro, Yeongtong-gu, Suwon, Gyeonggi-do 443-721, Korea Tel: +82-31-2195102, Fax: +82-31-2195999, E-mail: kjl@ajou.ac.kr 


\section{Introduction}

Gastroesophageal reflux disease (GERD) is common, and its prevalence is increasing in Asian countries including South Korea. $^{1-3}$ Based on the presence of erosions in the esophagus, GERD is classified into erosive reflux disease (ERD) and nonerosive reflux disease (NERD). NERD is known to be more common than ERD. When there is no evidence of ERD, symptoms may or may not be related to GERD. The ambulatory 24-hour esophageal $\mathrm{pH}$-impedance monitoring can be used to confirm the presence of pathological gastroesophageal acid or non-acid reflux and the causal relationship between symptoms and reflux events in patients who are suspected to have NERD. Based on the Rome IV criteria, the presence of symptoms including heartburn or chest pain, normal endoscopy, absence of eosinophilic esophagitis or major esophageal motor disorders as the etiology of symptoms, positive symptom association with reflux events, and absence of pathological acid or nonacid reflux on $\mathrm{pH}$-impedance monitoring suggest reflux hypersensitivity $(\mathrm{RH}) .{ }^{4}$ Although the gold standard for diagnosing GERD is considered to be 24-hour esophageal pH-impedance monitoring, it has disadvantages including inconvenience, timeconsuming, and the possibility of false negative results. It only detects reflux activity occurring during the test period.

Intra-luminal baseline impedance measurement in the esophagus can be used to evaluate changes in the integrity of the esophageal mucosa. Increased paracellular permeability of the esophageal mucosa can result in the alteration of mucosal impedance. Actually, lower intra-luminal baseline impedance levels (BILs) have been suggested to be evidence of GERD. ${ }^{5-7}$ The esophageal lumen is believed to be collapsed except in reflux and swallowing episodes, thus the intra-luminal BIL is most likely to be determined by the surrounding esophageal wall. Studies have shown that measurements of esophageal mucosal impedance can detect GERD. ${ }^{5-8}$ However, the cut-off value of esophageal baseline impedance for diagnosing GERD is not clearly established in Korean patients with esophageal or supraesophageal symptoms. Even, the cut-off values reported in previous studies are different. ${ }^{7-12}$ BILs were measured during night times in most of previous reports. ${ }^{7-12}$ The proper time for measurement of esophageal BILs has not been clearly established.

Thus, the aim of this study is to investigate the usefulness of esophageal BILs for the diagnosis of NERD and the proper time for measurement in endoscopy-negative Korean patients with esophageal or supraesophageal symptoms.

\section{Materials and Methods}

\section{Subjects}

Consecutive endoscopy-negative patients with esophageal (heartburn and chest pain) or supraesophageal (globus and cough) symptoms who underwent 24-hour esophageal multichannel intraluminal impedance-pH (MII-pH) monitoring were eligible for inclusion in this study. Their MII-pH data were analyzed. Patients were excluded if they were taking medications which could influence esophageal motor function or gastric acid secretion, or if they had erosive or ulcerative esophagitis on endoscopy performed during the medication (proton pump inhibitors [PPIs] and histamine $\mathrm{H} 2$ receptor antagonists)-off period. Patients with a history of upper gastrointestinal surgery, patients with eosinophilic esophagitis, or patients with major esophageal motility disorders were excluded. This study protocol was approved by the Institutional Review Board of Ajou University Hospital (AJIRB-MED-MDB-19-426).

\section{Twenty-four Hour Multichannel Intraluminal Impedance-pH Monitoring}

MII-pH monitoring was performed in the fasting state at night after at least a week of washout of gastric acid suppressive or motility agents. The position of the lower esophageal sphincter (LES) was determined using esophageal manometry. Subsequently, the $\mathrm{pH}$ electrode was located at $5 \mathrm{~cm}$ above the upper margin of the LES, and segments measuring intraluminal impedance were positioned at 3, 5, 7, 9, 15, and $17 \mathrm{~cm}$ above the LES. Patients were asked to maintain normal activities and eat their usual meals at their normal times. They were encouraged to keep a diary in which they recorded the times for meals, sleep, postural changes, and symptoms. Data was analyzed using Bio-View Analysis software (Sandhill Scientific Inc, Highlands Ranch, CO, USA), and manual analysis was done particularly for impedance data.

An acid reflux episode was defined as a drop in $\mathrm{pH}$ to less than 4.0, and pathological acid reflux was defined as the percentage of time with an acid reflux episode above $4.2 \%$. A reflux episode was defined as a retrograde drop in impedance by more than $50.0 \%$ of the baseline in the distal 2 consecutive channels, and pathological nonacid reflux was defined as the percentage of time with a reflux episode above $1.4 \%$ in the absence of pathological acid reflux. ${ }^{13,14}$ The symptom index $\geq 50 \%$ and/or symptom association probability $\geq 95 \%$ was considered positive for symptom correlation. ${ }^{14,15}$

Baseline impedance was measured in the proximal esophagus 
(the mean value of $15 \mathrm{~cm}$ and $17 \mathrm{~cm}$ channels) and distal esophagus (the mean value of $3 \mathrm{~cm}$ and $5 \mathrm{~cm}$ channels) around 10 minutes before meal ingestion, 10 minutes after meal ingestion, 30 minutes after meal ingestion, 30 minutes before the start of nighttime sleep, 30 minutes after the start of nighttime sleep, and 60 minutes after the start of nighttime sleep. Each single baseline value represents the average value of a 5-minute period around each time point avoiding swallows, refluxes, and $\mathrm{pH}$ drops. ${ }^{14,15} \mathrm{MII}-\mathrm{pH}$ monitoring data were reviewed manually by investigators (Y.G.K. and K.J.L.) blinded for the information of the patients.

\section{Statistical Methods}

The subjects were classified into 3 groups as follows: (1) the NERD group, defined as the presence of pathological acid and/or nonacid reflux; (2) the RH group, defined as positive for symptom correlation in the absence of pathological acid or nonacid reflux; and (3) the functional group, defined as the absence of pathological acid or nonacid reflux and negative for symptom correlation.

Data are expressed as means $\pm \mathrm{SD}$. Continuous variables were compared using Student's t test or ANOVA with post hoc test. The chi-square test was applied to compare categorical variables between groups. Receiver operating characteristic (ROC) curves were used to determine the cut-off value with the maximal sum of sensitivity and specificity. Statistical analyses were performed using the SPSS version 23.0 software package (SPSS; IBM Corp, Armonk, NY, USA), which calculates the area under the curve and $95 \%$ confidence intervals (CIs). A $P$-value $<0.05$ was considered to be significant.

\section{Results}

\section{Demographics and Characteristics of the Subjects}

A total of 104 patients (57 males and 47 females) were included in the study. The mean age of subjects was $47.9 \pm 13.9$ years. Chief complaints included esophageal symptoms such as heartburn (39 patients, $37.5 \%$ ) and chest pain (28 patients, 26.9\%), and supraesophageal symptoms such as globus and/or cough (37 patients, $35.5 \%)$. The baseline characteristics of the subjects are summarized in Table 1. The prevalence of pathological acid exposure and $\mathrm{RH}$ were significantly higher in the heartburn group, compared with the globus and/or cough group.

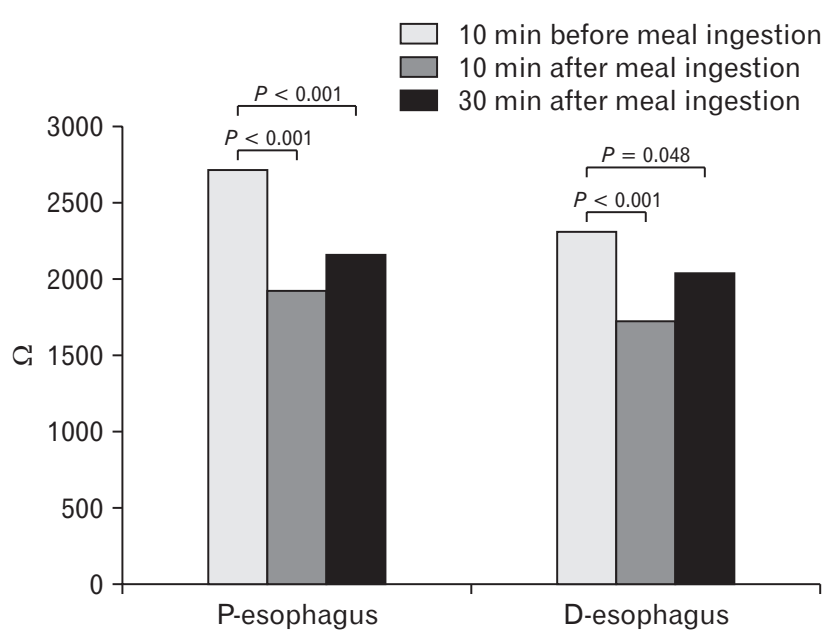

Figure 1. Changes of esophageal baseline impedance levels after meal ingestion. $\mathrm{P}$, proximal; $\mathrm{D}$, distal.

Table 1. Baseline Characteristics of the Subjects According to Chief Complaints

\begin{tabular}{lccc}
\hline \multicolumn{1}{c}{ Characteristics } & $\begin{array}{c}\text { Heartburn } \\
(\mathrm{n}=39)\end{array}$ & $\begin{array}{c}\text { Chest pain } \\
(\mathrm{n}=28)\end{array}$ & $\begin{array}{c}\text { Globus and/or cough } \\
(\mathrm{n}=37)\end{array}$ \\
\hline Gender & & & $19(51.4)$ \\
Male & $23(59.0)$ & $15(53.6)$ & $18(48.6)$ \\
Female & $16(41.0)$ & $13(46.4)$ & $46.9 \pm 13.5$ \\
Age (yr) & $46.8 \pm 14.5$ & $50.9 \pm 13.4$ & 0.229 \\
Pathological acid reflux & $14(35.9)^{\mathrm{a}}$ & $7(25.0)^{\mathrm{b}}$ & $13(35.1)$ \\
Pathological non-acid reflux & $6(15.4)$ & $7(25.0)$ & $1(2.7)$ \\
Reflux hypersensitivity & $9(23.1)^{\mathrm{c}}$ & $4(14.3)$ & 0.410 \\
\hline
\end{tabular}

${ }^{\mathrm{a}} \mathrm{P}<0.001,{ }^{\mathrm{b}} \mathrm{P}<0.01$, and ${ }^{\mathrm{c}} \mathrm{P}<0.05$, compared with globus by Pearson chi-square or Fisher's exact test, respectively.

Data are presented as number $(\%)$ or mean \pm SD. 


\section{Change of Esophageal Baseline Impedance Levels After Meal Ingestion or the Start of Nighttime Sleep}

Esophageal BILs around 10 minutes and 30 minutes after meal ingestion were significantly lower in the proximal $(P<0.001)$ and distal esophagus $(P<0.001)$, compared with those around 10 minutes before meal ingestion (Fig. 1).

Significantly lower esophageal BILs were observed around 30 minutes $(P<0.005)$ and 60 minutes $(P<0.005)$ after the start of nighttime sleep in the proximal esophagus, compared with those around 30 minutes before the start of nighttime sleep, which was not observed in the distal esophagus (Fig. 2).

\section{Comparison of Esophageal Baseline Impedance Levels in the Diagnostic Groups}

The BILs of the proximal esophagus did not significantly differ

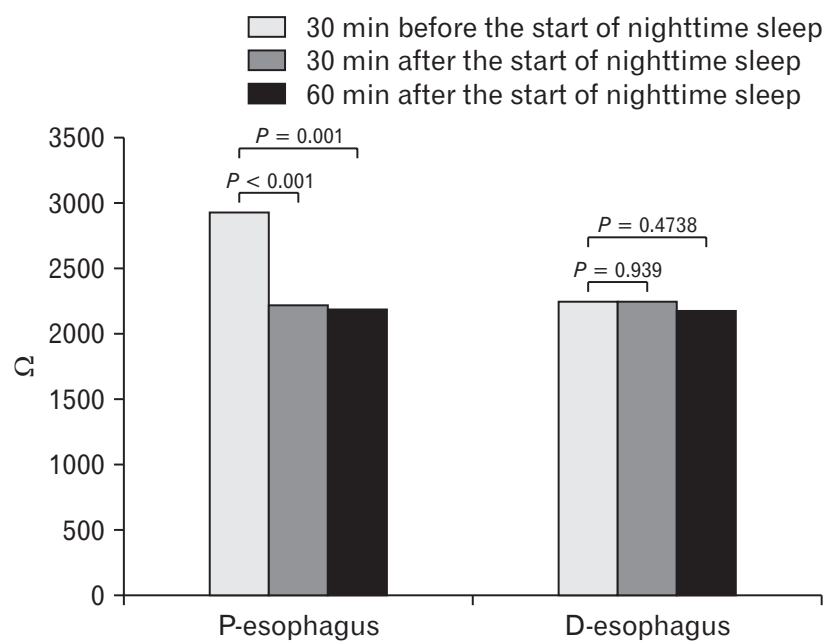

Figure 2. Changes of esophageal baseline impedance levels after the start of nighttime sleep. $\mathrm{P}$, proximal; $\mathrm{D}$, distal. at all time points among the NERD, $\mathrm{RH}$, and functional groups (Table 2). The BILs of the distal esophagus were significantly lower at all time points in the NERD group, but not in the RH group, compared with the functional group (Table 3).

\section{The Area Under the Receiver Operating Characteristic Curve for the Diagnosis of Nonerosive Reflux Disease and Reflux Hypersensitivity}

The area under the ROC curve for the diagnosis of NERD was significant at all time points and the highest around 30 minutes before and after the start of nighttime sleep. The area under the ROC curve for the diagnosis of $\mathrm{RH}$ was not significant at all time points (Table 4).

The area under the ROC curve for the diagnosis of NERD in patients with esophageal symptoms was the highest around $30 \mathrm{~min}-$ utes before the start of nighttime sleep $(0.821$ [95\% CI, 0.704-0.938,

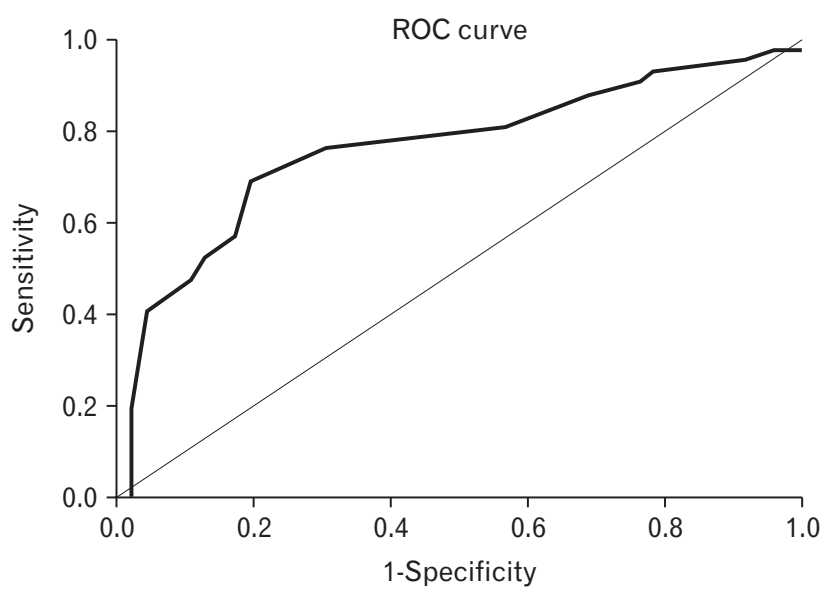

Figure 3. The receiver operating characteristic (ROC) curve for the diagnosis of nonerosive esophageal reflux disease in patients with esophageal or supraesophageal symptoms.

Table 2. Baseline Impedance Levels of the Proximal Esophagus in the Diagnostic Groups

\begin{tabular}{|c|c|c|c|c|}
\hline \multirow{2}{*}{ Time } & \multicolumn{4}{|c|}{ Impedance $(\Omega)$} \\
\hline & NERD group $(\mathrm{n}=42)$ & $\mathrm{RH}$ group $(\mathrm{n}=16)$ & Functional group $(n=46)$ & $P$-value \\
\hline 10 min before meal & $2760 \pm 1110$ & $2540 \pm 610$ & $2750 \pm 890$ & 0.720 \\
\hline $10 \mathrm{~min}$ after meal & $1860 \pm 820$ & $1870 \pm 930$ & $2010 \pm 970$ & 0.691 \\
\hline 30 min after meal & $2190 \pm 870$ & $2200 \pm 860$ & $2110 \pm 980$ & 0.911 \\
\hline $30 \mathrm{~min}$ before sleep onset & $3010 \pm 1180$ & $3070 \pm 640$ & $2830 \pm 1190$ & 0.658 \\
\hline 30 min after sleep onset & $2220 \pm 1170$ & $2510 \pm 800$ & $2140 \pm 960$ & 0.472 \\
\hline 60 min after sleep onset & $2190 \pm 1130$ & $2700 \pm 1020$ & $2020 \pm 830$ & 0.069 \\
\hline
\end{tabular}

NERD, nonerosive reflux disease; RH, reflux hypersensitivity.

Data are presented as mean $\pm \mathrm{SD}$. 
Table 3. Baseline Impedance Levels of the Distal Esophagus in the Diagnostic Groups

\begin{tabular}{lcccc}
\hline \multirow{2}{*}{ Time } & \multicolumn{3}{c}{ Impedance $(\Omega)$} \\
\cline { 2 - 5 } & NERD group $(\mathrm{n}=42)$ & RH group $(\mathrm{n}=16)$ & Functional group $(\mathrm{n}=46)$ & $P$-value \\
\hline 10 min before meal & $1890 \pm 940^{\mathrm{a}}$ & $2730 \pm 900$ & $2550 \pm 970$ & 0.001 \\
10 min after meal & $1310 \pm 690^{\mathrm{a}}$ & $2070 \pm 1010$ & $1990 \pm 1000$ & 0.001 \\
30 min after meal & $1460 \pm 690^{\mathrm{b}}$ & $2340 \pm 720$ & $2570 \pm 80 \pm 0$ & 0.004 \\
30 min before sleep onset & $1760 \pm 1180^{\mathrm{a}}$ & $2640 \pm 1070$ & $2580 \pm 900$ & 0.001 \\
30 min after sleep onset & $1670 \pm 930^{\mathrm{c}}$ & $2790 \pm 880$ & $2460 \pm 840$ & $<0.001$ \\
60 min after sleep onset & $1690 \pm 830^{\mathrm{c}}$ & $2700 \pm 960$ & & $<0.001$ \\
\hline
\end{tabular}

NERD, nonerosive reflux disease; RH, reflux hypersensitivity.

${ }^{a} P<0.005,{ }^{b} P<0.05$, and ${ }^{c} P<0.001$, compared with the functional group by Student's $t$ test or ANOVA with post hoc test.

Data are presented as mean $\pm \mathrm{SD}$.

Table 4. The Area Under the Receiver Operating Characteristic Curve for the Diagnosis of Nonerosive Reflux Disease and Reflux Hypersensitivity

\begin{tabular}{lcccc}
\hline \multirow{2}{*}{ Time } & \multicolumn{3}{c}{ AUC $(95 \% \mathrm{CI})$} \\
\cline { 2 - 5 } & NERD $(\mathrm{n}=42)$ & $P$-value & RH $(\mathrm{n}=16)$ & $0.433(0.273-0.592)$ \\
\hline 10 min before meal & $0.716(0.608-0.824)$ & $<0.001$ & $0.473(0.309-0.637)$ & 0.426 \\
10 min after meal & $0.712(0.605-0.819)$ & $<0.001$ & $0.446(0.293-0.599)$ & 0.748 \\
30 min after meal & $0.747(0.646-0.849)$ & $<0.001$ & $0.457(0.274-0.640)$ & 0.612 \\
30 min before sleep onset & $0.763(0.659-0.867)$ & $<0.001$ & $0.414(0.250-0.578)$ & 0.307 \\
30 min after sleep onset & $0.764(0.662-0.866)$ & $<0.001$ & $0.447(0.267-0.627)$ & 0.530 \\
60 min after sleep onset & $0.747(0.644-0.850)$ & &
\end{tabular}

AUC, area under the curve; NERD, nonerosive reflux disease; RH, reflux hypersensitivity.

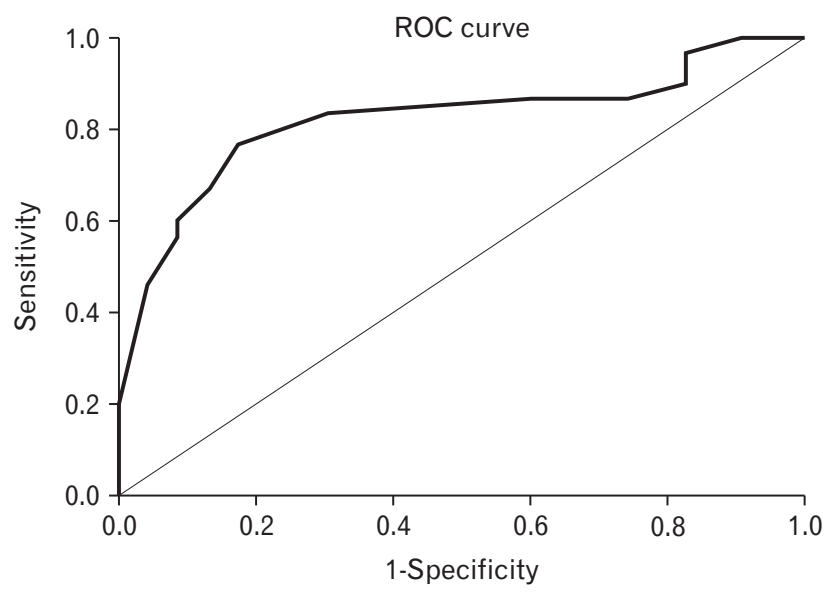

Figure 4. The receiver operating characteristic (ROC) curve for the diagnosis of nonerosive esophageal reflux disease in patients with esophageal symptoms.

$P<0.001])$. The area under the ROC curve for the diagnosis of NERD in patients with supraesophageal symptoms was the highest around 60 minutes after the start of nighttime sleep $(0.772$ [95\%
CI, $0.608-0.936, P=0.009])$.

\section{Cut-off Values for the Diagnosis of Nonerosive Reflux Disease}

When a cut-off value of $2375 \Omega$ was selected around 30 minutes before the start of nighttime sleep in patients with esophageal or supraesophageal symptoms, the sensitivity and specificity for the diagnosis of NERD were 0.762 and 0.696 , respectively (Fig. 3). When a cut-off value of $2375 \Omega$ was selected around 30 minutes before the start of nighttime sleep in patients with esophageal symptoms, the sensitivity and specificity for the diagnosis of NERD were 0.833 and 0.696 , respectively (Fig. 4). When a cut-off value of $2125 \Omega$ was selected around 60 minutes after the start of nighttime sleep in patients with supraesophageal symptoms, the sensitivity and specificity for the diagnosis of NERD were 0.833 and 0.739 , respectively (Fig. 5).

\section{Discussion}

The BILs of the distal esophagus measured during the ambu- 


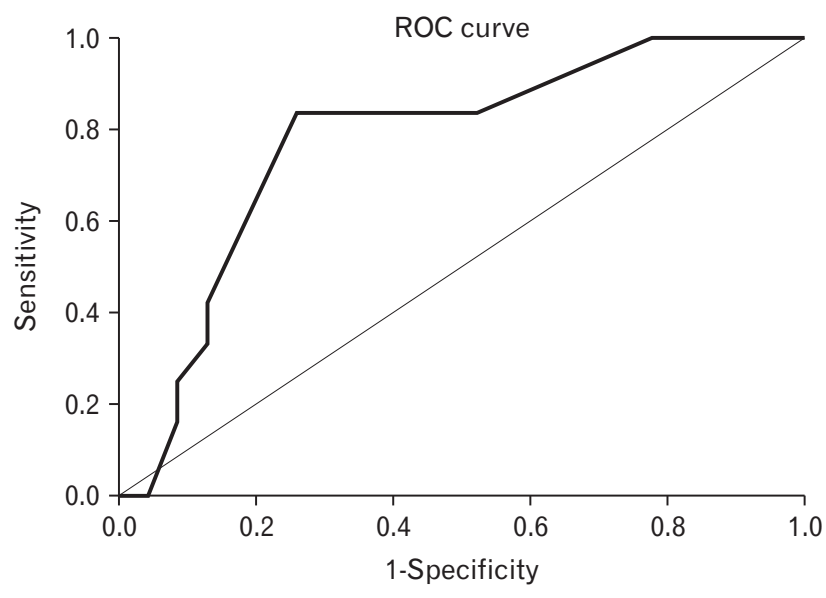

Figure 5. The receiver operating characteristic (ROC) curve for the diagnosis of nonerosive esophageal reflux disease in patients with supraesophageal symptoms.

latory 24-hour esophageal $\mathrm{pH}$-impedance monitoring are considered to reflect esophageal reflux burden. Mean nocturnal baseline impedance has been negatively correlated with esophageal acid exposure time, and suggested to be a predictor of symptomatic improvement following anti-reflux therapy. ${ }^{16}$ The results of the present study indicated that BILs of the distal esophagus can be useful for the diagnosis NERD in endoscopy-negative Korean patients with esophageal or supraesophageal symptoms, but not for the diagnosis of RH.

PPI therapy is regarded as the mainstay of pharmacologic treatment for GERD, and can also be used for the diagnosis of GERD in clinical practice. In patients with GERD-like symptoms, unsatisfactory response to a PPI trial represents the requirement of ambulatory esophageal $\mathrm{pH}$-impedance monitoring to confirm GERD. Although ambulatory $\mathrm{pH}$-impedance monitoring is considered to be the gold standard test for the measurement of reflux activity, it is not likely to be sufficient in the diagnosis of GERD. It has many disadvantages, and the diagnostic sensitivity for GERD is limited. Actually, in the Lyon consensus, the diagnosis of GERD needs a higher value of esophageal acid exposure time. We defined pathological acid reflux as the percentage of time with an acid reflux episode above $4.2 \%$. The use of the Lyon consensus criteria remarkably reduces the number of patients with pathological acid reflux from 21 patients (20.2\%) to 9 patients (8.6\%). So, the use of the Lyon consensus criteria may reduce diagnostic yield for GERD, particularly for NERD, because acid reflux burden in NERD patients is considered to be lower than that in ERD patients.

Recently, several studies suggest that mean nocturnal BILs of the distal esophagus and post-reflux swallow-induced peristaltic wave can increase the diagnostic yield of ambulatory esophageal pH-impedance monitoring. ${ }^{8,15-18}$ Abnormally low esophageal mean nocturnal baseline impedance is suggested to be an additional marker of GERD. Actually, esophageal BILs have been recently reported to be correlated with response of symptoms to antireflux therapy. ${ }^{19}$ Our findings of the present study suggest that low BILs of the distal esophagus measured around 30 minutes before the start of nighttime sleep was a marker of NERD in patients with esophageal symptoms such as heartburn or chest pain, whereas low BILs of the distal esophagus measured around 60 minutes after the start of nighttime sleep was a marker of NERD in patients with supraesophageal symptoms such as globus and/or cough. When considering this finding, supraesophageal NERD symptoms might be more associated with nighttime recumbent position than esophageal NERD symptoms.

Esophageal BILs are measured from the most distal impedance channel during the nighttime recumbent period. Esophageal impedance levels may fluctuate within a day. In previous studies, mean nocturnal baseline impedance was measured during the overnight rest for a 10-minute period at around 3 time points $(1,2$, and $3 \mathrm{AM}$ ), avoiding refluxes and swallows. ${ }^{10-18}$ In the present study, we found that BILs of the distal and proximal esophagus were decreased after meal ingestion. It is assumed that increased reflux or swallowing associated with meal ingestion plays a role in decreasing BILs of the esophagus. The BILs of the proximal esophagus were significantly altered during the nighttime recumbent period, but those of the distal esophagus were not. These observations can provide evidence that the period before or after the start of nighttime sleep is appropriate to assess BILs of the distal esophagus for the diagnosis of NERD. Our findings showed that 2 time points (30 minutes before and 60 minutes after the start of nighttime sleep) were most appropriate, and represented the highest value of area under the ROC curve for the diagnosis of NERD.

In the present study, we showed that BILs of the distal esophagus were significantly lower at all time points in the NERD group, compared with the functional group. Whereas, the BILs of the proximal esophagus did not significantly differ at all time points among the diagnostic groups. These findings indicate that BILs of the distal esophagus may be useful for differentiating NERD and functional heartburn. However, we failed to demonstrate that measurement of BILs is useful for differentiating $\mathrm{RH}$ and functional heartburn. Low BILs of the esophagus may be associated with high reflux burden or increased esophageal acid exposure. Kessing et $\mathrm{al}^{19}$ suggest that baseline impedance is related to esophageal 
acid exposure, and could be a marker of reflux-induced changes in the esophageal mucosa. This is in line with previous observations showing that the acid exposure affects the baseline impedance. ${ }^{20}$ Similarly, higher basal impedance levels are found to be related to functional heartburn. ${ }^{13}$

There may be discrepancy between studies regarding the cut-off value for the diagnosis of GERD. The cut-off levels of $2292 \Omega$ and $2100 \Omega$ for differentiation between NERD and functional heartburn have been reported. ${ }^{11,12}$ Whereas, the cut-off level of $1582 \Omega$ for the diagnosis of GERD was also reported in another study. 9 The present study demonstrated that the cut-off value of $2375 \Omega$ or $2125 \Omega$ was useful for the diagnosis of NERD in patients with esophageal or supraesophageal symptoms. When the cut-off value of $2375 \Omega$ was selected around 30 minutes before the start of nighttime sleep in patients with esophageal symptoms, the sensitivity and specificity for the diagnosis of NERD were 0.833 and 0.696 , respectively. When the cut-off value of $2125 \Omega$ was selected around 60 minutes after the start of nighttime sleep in patients with supraesophageal symptoms, the sensitivity and specificity for the diagnosis of NERD were 0.833 and 0.739 , respectively.

In conclusion, distal and proximal esophageal BILs can be altered by meal ingestion. The BILs of the distal esophagus measured at time points around 30 minutes before or 60 minutes after the start of nighttime sleep appear to be useful for the diagnosis of NERD, but not for the diagnosis of RH, in endoscopy-negative Korean patients with esophageal or supraesophageal symptoms.

Financial support: This research was supported by a grant of Patient-Centered Clinical Research Coordinating Center funded by the Ministry of Health \& Welfare, Republic of Korea (Grant No. HI19C0481, HC19C0060).

\section{Conflicts of interest: None.}

Author contributions: Young-Gun Kim and Kwang Jae Lee contributed to study design and data collection; Young-Gun Kim, Choong-Kyun Noh, and Kwang Jae Lee analyzed data, wrote the manuscript, and evaluated the eligibility of subjects; and Kwang Jae Lee provided critical revision.

\section{References}

1. Lee SY, Lee KJ, Kim SJ, Cho SW. Prevalence and risk factors for overlaps between gastroesophageal reflux disease, dyspepsia, and irritable bowel syndrome: a population-based study. Digestion 2009;79:196-201.

2. Min BH, Huh KC, Jung HK, et al. Prevalence of uninvestigated dys- pepsia and gastroesophageal reflux disease in Korea: a population-based study using the Rome III criteria. Dig Dis Sci 2014;59:2721-2729.

3. Shim KN, Hong SJ, Sung JK, et al. Clinical spectrum of reflux esophagitis among 25,536 Koreans who underwent a health check-up: a nationwide multicenter prospective, endoscopy-based study. J Clin Gastroenterol 2009;43:632-638.

4. Aziz Q, Fass R, Gyawali CP, Miwa H, Pandolfino JE, Zerbib F. Functional esophageal disorders. Gastroenterology 2016;150:1368-1379.

5. Katzka DA, Ravi K, Geno DM, et al. Endoscopic mucosal impedance measurements correlate with eosinophilia and dilation of intercellular spaces in patients with eosinophilic esophagitis. Clin Gastroenterol Hepatol 2015;13:1242-1248, e1.

6. Yuksel ES, Higginbotham T, Slaughter JC, et al. Use of direct, endoscopic-guided measurements of mucosal impedance in diagnosis of gastroesophageal reflux disease. Clin Gastroenterol Hepatol 2012;10:11101116.

7. Ates F, Yuksel ES, Higginbotham T, et al. Mucosal impedance discriminates GERD from non-GERD conditions. Gastroenterology 2015;148:334-343.

8. Sun YM, Gao Y, Gao F. Role of esophageal mean nocturnal baseline impedance and post-reflux swallow-induced peristaltic wave index in discriminating Chinese patients with heartburn. J Neurogastroenterol Motil 2019;25:515-520.

9. Ravi K, Geno DM, Vela MF, Crowell MD, Katzka DA. Baseline impedance measured during high-resolution esophageal impedance manometry reliably discriminates GERD patients. Neurogastroenterol Motil 2017;29:e12974.

10. Martinucci I, de Bortoli N, Savarino E, et al. Esophageal baseline impedance levels in patients with pathophysiological characteristics of functional heartburn. Neurogastroenterol Motil 2014;26:546-555.

11. Frazzoni M, Savarino E, de Bortoli N, et al. Analyses of the post-reflux swallow-induced peristaltic wave index and nocturnal baseline impedance parameters increase the diagnostic yield of impedance-pH monitoring of patients with reflux disease. Clin Gastroenterol Hepatol 2016;14:40-46.

12. Kandulski A, Weigt J, Caro C, Jechorek D, Wex T, Malfertheiner P. Esophageal intraluminal baseline impedance differentiates gastroesophageal reflux disease from functional heartburn. Clin Gastroenterol Hepatol 2015;13:1075-1081.

13. Kushnir VM, Sathyamurthy A, Drapekin J, Gaddam S, Sayuk GS, Gyawali CP. Assessment of concordance of symptom reflux association tests in ambulatory $\mathrm{pH}$ monitoring. Aliment Pharmacol Ther 2012;35:1080-1087.

14. Patel A, Sayuk GS, Gyawali CP. Parameters on esophageal pH-impedance monitoring that predict outcomes of patients with gastroesophageal reflux disease. Clin Gastroenterol Hepatol 2015;13:884-891.

15. Patel A, Wang D, Sainani N, Sayuk GS, Gyawali CP. Distal mean nocturnal baseline impedance on $\mathrm{pH}$-impedance monitoring predicts reflux burden and symptomatic outcome in gastro-oesophageal reflux disease. Aliment Pharmacol Ther 2016;44:890-898.

16. de Bortoli N, Martinucci I, Savarino E, et al. Association between baseline impedance values and response proton pump inhibitors in patients with heartburn. Clin Gastroenterol Hepatol 2015;13:1082-1088, e1. 
17. Frazzoni L, Frazzoni M, de Bortoli N, et al. Postreflux swallow-induced peristaltic wave index and nocturnal baseline impedance can link PPIresponsive heartburn to reflux better than acid exposure time. Neurogastroenterol Motil 2017;29:e13116.

18. Rengarajan A, Savarino E, Coletta MD, Ghisa M, Patel A, Gyawali P. Mean nocturnal baseline impedance correlates with symptom outcome when acid exposure time is inconclusive on esophageal reflux monitoring.
Clin Gastroenterol Hepatol 2020;18:589-595.

19. Kessing BF, Bredenoord AJ, Weijenborg PW, Hemmink GJ, Loots CM, Smout AJ. Esophageal acid exposure decreases intraluminal baseline impedance levels. Am J Gastroenterol 2011;106:2093-2097.

20. Farré R, Blondeau K, Clement D, et al. Evaluation of oesophageal mucosa integrity by the intraluminal impedance technique. Gut 2011;60:885892. 